\title{
Automatic processing of memory for spatial location
}

\author{
AMY L. SHADOIN and NORMAN R. ELLIS \\ University of Alabama, Tuscaloosa, Alabama
}

\begin{abstract}
Earlier research on the automaticity of memory for spatial location was extended. A series of four-photograph sets were presented to subjects, who then attempted to relocate the photographs to their original locations on posters. Number of locations on the posters varied from 4,9, or 16 , and instructions were intentional or incidental. Memory for location decreased with increased matrix size. Instructions had no effect. The recognition of photographs was unaffected by these variables. No firm evidence for effortful processing of location memory was found.
\end{abstract}

Hasher and Zacks $(1979,1984)$ theorized that spatial location, temporal order, and frequency of occurrence are automatically encoded into long-term memory. Their criteria for defining automatic processes were the following: (1) they occur without intention or awareness, (2) they are not improved with instruction, practice, or feedback, (3) they make minimal demands on central processing capacity, (4) they are not affected by alterations in attentional capacity due to stress, arousal, or mood, and (5) they are developmentally invariant across age and intelligence.

Empirical studies of memory for location prompted by Hasher and Zacks's (1979) automaticity theory have yielded mixed results. To date, most of the location memory research has used two experimental paradigms. One approach, used by Ellis and his associates (Dulaney \& Ellis, 1991; Ellis, 1990, 1991; Ellis, Katz, \& Williams, 1987; Ellis \& Rickard, 1989; Ellis, Woodley-Zanthos, \& Dulaney, 1989; Katz \& Ellis, 1991), is based on the Von Wright, Gebhart, and Karttunen (1975) procedure. Another approach, used by Naveh-Benjamin $(1987,1988)$, is the procedure developed by Mandler, Seegmiller, and Day (1977). Research generated with these two approaches has resulted in conflicting evidence on the automaticity issue. Ellis (1990, 1991), who finds support for Hasher and Zacks's position, has suggested that the discrepancy in findings may be due to task variables in the procedure used by Mandler et al. (1977) and Naveh-Benjamin (1987, 1988) that require effortful processing.

Von Wright et al.'s (1975) procedure involves the presentation of a series of pictures arranged in four-picture displays. Subjects are instructed to look at each of the pictures in the $2 \times 2$ matrices. This is followed by a test for location. Mandler et al.'s (1977) procedure involves the

\footnotetext{
This research was supported by Grant HD15558 to the second author from the National Institute of Child Health and Human Development. The first author is a Fellow in the Mental Retardation Training Program at the University of Alabama and is supported by Training Grant HD0762 from the National Institute of Child Health and Human Development. Reprint requests should be sent to Norman R. Ellis, Department of Psychology, University of Alabama, Box 870348, Tuscaloosa, AL 35487-0348.
}

presentation, in a single trial, of 16 objects arranged in a $6 \times 6$ matrix. The Naveh-Benjamin variant of this procedure uses 20 drawings of objects. In both procedures, subjects attempt to relocate pictures to their original positions in the matrix.

Evidence favorable to Hasher and Zacks's (1979) position has been gathered using the Ellis/Von Wright task. With this method, location memory is developmentally invariant, intellectually invariant, and resistant to improvement through instruction (Dulaney \& Ellis, 1991; Ellis et al., 1987; Ellis et al., 1989; Katz \& Ellis, 1991). Additional experiments have shown that memory for location is unaffected by practice, instruction, concurrent processing, and strategy manipulations (Ellis, 1990, 1991). Ellis and Rickard (1989) found that memory for location was relatively durable over a $24-\mathrm{h}$ retention interval. Taken together, the studies by Ellis and his associates are supportive of Hasher and Zacks's theory.

On the other hand, studies by Naveh-Benjamin (1987, 1988) have failed to find support for Hasher and Zacks's position. Using the paradigm developed by Mandler et al. (1977), Naveh-Benjamin found that location memory was affected by intention, subject age, and individual differences in intelligence and developmental age. He also reported evidence of improvement in relocation accuracy following practice and strategy training. Deterioration in relocation accuracy was found under conditions of concurrent processing demands. These results are inconsistent with Hasher and Zacks's (1979) automaticity criteria.

The Ellis paradigm and the Naveh-Benjamin paradigm differ with respect to their spatial complexity: the number of locations differ as well as the salience of the location cues, either of which may affect initial perception of locations. The tasks also may differ in the extent to which interference occurs. In the single-trial task, only one item appears in a location; in the multiple-trial task, many items appear in each location. The former also provides the opportunity for strategy use. This would not be feasible in the latter.

The present experiment was an attempt to determine whether or not the spatial complexity of the stimulus dis- 
play influenced memory for location. To minimize other potentially effortful processing aspects of the task, the multiple-trial format was used. Spatial complexity of the matrix was manipulated through variation in matrix size. The subjects received either intentional or incidental instructions. It was predicted that relocation accuracy would decrease with increases in matrix size. This decrease should be less for those receiving intentional instructions, assuming that subjects' attention to location cues may be facilitated by intent in the more complex tasks.

\section{Method}

Subjects. The 156 subjects were recruited from introductory psychology classes. They received course credit for participation. They were randomly and equally assigned to a $2 \times 3$ factorial design defined by two instructional groups and three matrix-size groups.

Materials. The three tasks included different-sized matrices. The $2 \times 2$ matrix was $17.5 \times 22.8 \mathrm{~cm}$, the $3 \times 3$ matrix was $27 \times 35.6 \mathrm{~cm}$, and the $4 \times 4$ matrix was $35 \times 46.4 \mathrm{~cm}$. White graphic tape was used on heavy black posterboard to construct matrices of either 4,9 , or 16 locations. The stimuli mounted in the center of each space consisted of a $6.35 \times 8.89 \mathrm{~cm}$ card with a colored line drawing of a common object (i.e., a comb, a desk, a car, etc.) against a white background. There were 31 posters for each task, and four line drawings were mounted on each poster. The same stimuli were used for the three tasks. The first and last three posters of each task were buffers; memory for these items was not tested.

Twenty-five line drawings were randomly selected as target items. Posters numbered 4 through 28 each contained one target item and three nontarget items. Buffer posters contained only nontarget items. Target items appeared approximately equally often in any given location. For the item-recognition task, the subjects attempted to sort a randomly selected 50-picture set into targets (25) and distractors (25).

Procedure. The subjects were told that this was an experiment in media advertising. All subjects were asked to study each poster and choose the picture most likely to be used in printed advertising and the picture least likely to be used in printed advertising. In the intentional condition, the subjects were also told they would be tested for location of pictures on the posters. In the incidental condition, there was no mention of a test for location. The subjects were allowed to study the posters at a self-paced rate, but they were not allowed to return to previously studied posters. Total study time was recorded.

After studying the posters, the subjects were given an item-recognition test. The relocation test followed, using only the recognized targets.

\section{Results}

Separate 2 (instruction) $\times 3$ (matrix size) analyses of variance (ANOVAs) were performed on the study-time (all trials combined), item-recognition, and item-relocation data. The $2 \times 3$ ANOVA on study time yielded significant differences for matrix size $[F(2,155)=7.19, p=$ .001 ]. Orthogonal contrasts showed the $4 \times 4$ matrix to be significantly different from the other two matrices $[t(153)=3.53, p<.001]$. Study times for the $2 \times 2$ and $3 \times 3$ matrices did not differ significantly from each other. There were no other significant effects involving study time. Table 1 provides mean total study time $\times$ matrix size and instructional condition.

The $2 \times 3$ ANOVA of item recognition revealed no significant differences in hits, false alarms, or $d^{\prime}$ due to instruction or matrix size. Table 1 presents means and standard deviations (SDs) for the item-recognition data.

A $2 \times 3$ ANOVA of item-relocation accuracies (corrected for chance responding) yielded significant differ-
Table 1

Means (Ms) and Standard Deviations (SDs) of Study Time and the Proportion of Pictures Recognized

\begin{tabular}{|c|c|c|c|c|c|c|c|c|}
\hline \multirow{2}{*}{$\begin{array}{c}\text { Matrix } \\
\text { Size }\end{array}$} & \multicolumn{2}{|c|}{$\begin{array}{l}\text { Study Time } \\
\text { (in Minutes) }\end{array}$} & \multicolumn{2}{|c|}{ Hits } & \multicolumn{2}{|c|}{$\begin{array}{c}\text { False } \\
\text { Alarms }\end{array}$} & \multicolumn{2}{|c|}{$d^{\prime}$} \\
\hline & $M$ & $S D$ & $\boldsymbol{M}$ & $S D$ & $\boldsymbol{M}$ & $S D$ & $\boldsymbol{M}$ & $S D$ \\
\hline \multicolumn{9}{|c|}{ Incidental Instruction } \\
\hline $\begin{array}{l}2 \times 2 \\
3 \times 3 \\
4 \times 4\end{array}$ & $\begin{array}{l}5.28 \\
5.42 \\
6.02\end{array}$ & $\begin{array}{l}1.40 \\
1.70 \\
1.56\end{array}$ & $\begin{array}{l}.92 \\
.89 \\
.90\end{array}$ & $\begin{array}{l}.07 \\
.11 \\
.08\end{array}$ & $\begin{array}{l}.06 \\
.06 \\
.06\end{array}$ & $\begin{array}{l}.06 \\
.07 \\
.07\end{array}$ & $\begin{array}{l}3.29 \\
3.10 \\
3.19\end{array}$ & $\begin{array}{l}.80 \\
.68 \\
.74\end{array}$ \\
\hline \multicolumn{9}{|c|}{ Intentional Instruction } \\
\hline $\begin{array}{l}2 \times 2 \\
3 \times 3 \\
4 \times 4\end{array}$ & $\begin{array}{l}4.92 \\
5.63 \\
6.43\end{array}$ & $\begin{array}{l}1.35 \\
1.32 \\
1.75\end{array}$ & $\begin{array}{l}.91 \\
.92 \\
.93\end{array}$ & $\begin{array}{l}.11 \\
.06 \\
.06\end{array}$ & $\begin{array}{l}.06 \\
.06 \\
.03\end{array}$ & $\begin{array}{l}.06 \\
.06 \\
.05\end{array}$ & $\begin{array}{l}3.25 \\
3.28 \\
3.61\end{array}$ & $\begin{array}{l}.74 \\
.81 \\
.63\end{array}$ \\
\hline
\end{tabular}

ences due to matrix size $[F(2,155)=18.06, p<.01]$. Orthogonal contrasts for matrix size showed relocation accuracy for the $4 \times 4$ matrix to be significantly different from relocation accuracy of the other two matrices $[t(153)$ $=5.61, p<.001]$. Relocation accuracies for the $2 \times 2$ and $3 \times 3$ matrices were significantly different from each other as well $[t(153)=2.07, p<.05]$. No effect was found for instruction. The interaction effect was nonsignificant. Means and $S D$ s for the relocation proportions are given in Table 2. The proportions were corrected for chance responding by subtracting .2500 from the $2 \times 2$ scores, .1111 from the $3 \times 3$ scores, and .0625 from the $4 \times 4$ scores.

Pearson correlation coefficients were computed for each of the matrix-size groups to assess the strength of the relationships between study time, $d^{\prime}$, and relocation accuracy. No significant relationships among these variables were found.

\section{Discussion}

These results confirm and extend earlier research by Ellis and his associates (Dulaney \& Ellis, 1991; Ellis, 1990, 1991; Ellis et al., 1987; Ellis \& Rickard, 1989; Ellis et al., 1989; Katz \& Ellis, 1991). As predicted, a matrix-size effect was found for item relocation but not for item recognition. Table 2 shows that relocation accuracy declined as matrix size increased, suggesting that the more complex matrices may affect perceptual aspects of processing. However, it is interesting that relocation accuracy, and not item recognition, is affected. As the number of locations increases, the salience of the location cues decreases, making discrimination of locations more effortful. The matrix-size effect tends to support the contention that the Mandler et al. (1977) and the Naveh-Benjamin $(1987,1988)$ tasks may contain subcomponents

Table 2

Means (Ms) and Standard Deviations (SDs) of the Proportions of Pictures Relocated

\begin{tabular}{ccc}
\hline Matrix Size & $M$ & $S D$ \\
\hline & Incidental Instruction & \\
$2 \times 2$ & .47 & .17 \\
$3 \times 3$ & .37 & .14 \\
$4 \times 4$ & .27 & .12 \\
& Intentional Instruction & \\
$2 \times 2$ & .44 & .15 \\
$3 \times 3$ & .43 & .14 \\
$4 \times 4$ & .31 & .11 \\
\hline
\end{tabular}


that require effortful processing. However, the failure to find an effect due to the different instructions is troublesome. Intentional instructions should have facilitated the processing of the more complex matrices, since they make heavier demands on effortful processing. Table 2 shows a trend in this direction, but it falls short of statistical significance. The study-time data also tend to support the view that more effortful processing is required by the more complex matrices, but intentional instructions were not associated with longer study time.

The failure to find an interaction between matrix size and instruction is probably due to the multiple-trial procedure, which provides the occasion for strong interference effects and a lack of opportunity for strategic processing. Many different pictures are presented in each location, and the test for location is delayed. This contrasts with the single-trial procedure in which only one picture is presented in a location and the location test follows immediately. Even though memory for location is less accurate in the perceptually more complex task, we cannot link this to effortful processing in the present study, because we could not show an instruction effect in the more complex task. This is not entirely ruled out, however, since there is a trend in the expected direction. Since memory for location was affected by matrix size and picture recognition was not, it seems likely that location cues are less salient under these conditions and that some locations are not perceived.

In keeping with Hasher and Zacks's (1979) position, these findings provide no firm evidence of effortful processing in memory for location. A study varying matrix size and instruction, using the single-trial procedure, is needed to determine if effortful processing is involved in the single-trial procedure used by Mandler et al. (1977) and NavehRenjamin $(1987,1988)$.

\section{REFERENCES}

Dulaney, C. L., \& ElLIS, N. R. (1991). Long-term recognition memory for items and attributes by retarded and nonretarded persons. Intelligence, 15, 105-115.
ElLIS, N. R. (1990). Is memory for spatial location automatically encoded? Memory \& Cognition, 18, 584-592.

ElLIS, N. R. (1991). Automatic and effortful processes in memory for spatial location. Bulletin of the Psychonomic Society, 29, 28-30.

Eluis, N. R., Katz, E., \& Williams, J. E. (1987). Developmental aspects of memory for spatial location. Journal of Experimental Child Psychology, 44, 401-412.

Ellis, N. R., \& RICKARD, T. C. (1989). The retention of automatically and effortfully encoded stimulus attributes. Bulletin of the Psychonomic Society, 27, 299-302.

Ellis, N. R., Woodley-Zanthos, P., \& Dulaney, C. L. (1989). Memory for spatial location in children, adults, and mentally retarded persons. American Journal on Mental Retardation, 93, 521-527.

HASHER, L., \& ZACKS, R. T. (1979). Automatic and effortful processes in memory. Journal of Experimental Psychology: General, 108, 356-388.

HASHER, L., \& ZACKS, R. T. (1984). Automatic processing of fundamental information: The case of frequency of occurrence. American Psychologist, 39, 1372-1388.

KatZ, E. R., Eluis, N. R. (1991). Memory for spatial location in retarded and nonretarded persons. Journal of Mental Deficiency Research, 35, 209-220.

Mandler, J. M., Seegmiller, D., \& Day, J. (1977). On the coding of spatial information. Memory \& Cognition, 5, 10-16.

Naveh-Benjamin, M. (1987). Coding of spatial location information: An automatic process? Journal of Experimental Psychology: Learning, Memory, \& Cognition, 13, 595-605.

NAVEh-Benjamin, M. (1988). Recognition memory of spatial location information: Another failure to support automaticity. Memory \& Cog nition, 16, 437-445.

Von Wright, J. M., Gebhard, P., \& Karttunen, M. (1975). A developmental study of the recall of spatial location. Journal of Experimental Child Psychology, 20, 181-190. 